\title{
Validade de Conteúdo do Instrumento para Avaliação do Conhecimento Tático Processual no Basquetebol
}

\section{Content validity of the instrument for assessment of the tactical procedural knowledge in basketball}

\author{
Juan C. P. Morales, Pablo J. Greco \& Ricardo L. Andrade
}

\begin{abstract}
PALAVRAS-CHAVE: Basquetebol, coeficiente de validade de conteúdo, conhecimento tático processual.

RESUMO: objetivou-se estabelecer a validade de conteúdo do teste de conhecimento tático processual para basquetebol (TCTP-Bb), a partir do cálculo do coeficiente de validade de conteúdo (CVC) proposto por Hernandez-Nieto (2002). Participaram do estudo sete técnicos de basquetebol ( $\mathrm{n}=07)$ formados em educação física e com experiência mínima de dez anos atuando especificamente no processo de iniciação esportiva, especialização e alto nível de rendimento na modalidade. Propuseram-se 24 itens para avaliar o comportamento tático - técnico individual e coletivo (tática de grupo) do jogador no ataque (com e sem bola), e do jogador na defesa (marcando ao jogador com bola e sem bola). Foi calculado o coeficiente de validade de conteúdo (CVC), a partir da clareza da linguagem e da pertinência prática, para cada item do instrumento $\left(\mathrm{CVC}_{\mathrm{c}}\right)$ e para o instrumento como um todo $\left(\mathrm{CVC}_{\mathrm{t}}\right)$. Os resultados confirmam que para clareza da linguagem $\left(\mathrm{CVC}_{\mathrm{t}}=\right.$ $0,94)$ e para pertinência prática $\left(\mathrm{CVC}_{\mathrm{t}}=0,91\right)$, os itens propostos apresentam propriedades psicométricas satisfatórias.

KEY-WORDS: Basketball, content validity coefficient, tactical procedural
\end{abstract}

\section{knowledge.}

ABSTRACT: The aim of this study was to establish the content validity of the tactical procedural knowledge test in basketball (TPKT-Bb), starting from the calculation of the content validity coefficient (CVC) as proposed by Hernandez-Nieto (2002). Seven basketball coaches $(n=07)$ participated in the study. All of them were graduated in Physical Education and had a minimum of ten years experience in working with the processes of sports initiation, specialization and high performance in basketball. We have proposed 24 items to evaluate the behaviour of the players during individual and group tactical actions. During offense situations, the player was assessed when in possession of the ball and also when not. In the same way, on defense the player was evaluated when marking the ball-handler and also when marking the opponent not in possession of the ball. The content validity coefficient (CVC) was calculated by means of language clearness and practical relevance for each item of the instrument $\left(\mathrm{CVC}_{\mathrm{c}}\right)$ and for the overall instrument $\left(\mathrm{CVC}_{\mathrm{t}}\right)$. The results confirm that, for the purposes of language clearness $\left(\mathrm{CVC}_{\mathrm{t}}=0,94\right)$ and practical relevance $\left(\mathrm{CVC}_{\mathrm{t}}=0,91\right)$, the proposed items present satisfactory psychometric properties.

\section{Introdução}

O processo de análise e avaliação da ação esportiva no basquetebol tem considerado o conhecimento tático processual (CTP), como uma das variáveis que oportuniza a adequada descrição do desempenho do atleta. O CTP é definido como a capacidade do atleta de agir selecionando o gesto técnico mais adequado, conforme a situação - problema da competição que defronta (GRECO; MEMMERT; MORALES, 2010; RODRIGUES, 2001). Nesse contexto, o sucesso ou fracasso da ação esportiva depende também do nível de desenvolvimento do CTP do atleta.

Para Nitsch (2009) a ação esportiva se concretiza na interação entre o processo de percepção da situação, diferenciando suas oportunidades e restrições. Nesse processo, a memória, atenção, concentração, antecipação e pensamento,

Endereço para correspondência: Prof. Ms. Juan Carlos Pérez Morales, Escola de Educação Física, Fisioterapia e Terapia Ocupacional da UFMG. Centro de Excelência Esportiva - CENESP, Grupo Estudos de Cognição e Açâo - GECA. Av. Presidente Antônio Carlos, 6627- Pampulha. Belo Horizonte-MG. Cep: 31.310-250.

E-mail: jkperezmorales@gmail.com oportunizam a seleção da resposta tática e sua concretização motora via um gesto técnico adequado.

Nesse contexto, toda ação nos jogos esportivos coletivos (JEC) é uma ação tática que contempla pressupostos cognitivos indispensáveis à regulação dessa ação (GARGANTA, 2004). A cognição se constitui de um conjunto de processos (por exemplo, percepção, atenção, pensamento, memória, tomada de decisão, entre outros) que interagem na aquisição e desenvolvimento do conhecimento tático necessário à solução e execução motora das diferentes tarefas ou problemas do jogo (GRECO, 1999).

Conforme Sève, Saury, Leblanc e Durand (2005), existem duas linhas de pesquisa que estudam as habilidades cognitivas no esporte e especificamente nos JEC. A primeira linha de pesquisa se centra nas características do conhecimento tático do atleta e sua adequada utilização na situação esportiva. A principal hipótese nessa linha de pesquisa é que o atleta perito utiliza com mais qualidade seu conhecimento na soluçáo dos problemas que defronta na competição (SÈVE; SAURY; LEBLANC; DURAND, 2005). A segunda linha de pesquisa estuda o processamento da informação e o processo de toma- 
da de decisão do atleta.

Essas linhas de pesquisa analisam dois componentes da atividade esportiva: a) a ação pela ativação do conhecimento e b) a ação em relação aos eventos provenientes da situação. Diferentes instrumentos têm sido desenvolvidos (FRENCH; THOMAS, 1987; GRÉHAIGNE; GODBOUT; BOUTHIER, 1997; 2001; OSLIN; MITCHELL; GRIFFIN, 1998; MEMMERT, 2002; TALLIR, et. al., 2003; COSTA, et. al., 2011), para identificar comportamentos e níveis de desempenho entre jogadores peritos e novatos; oportunizando a analise da eficácia dos métodos de ensino-aprendizagem (formais e incidentais) centrados na tática dos JEC.

No caso específico do basquetebol, diferentes estudos (ALLISON; THORPE, 1997; BOUTMANS, 1983; IGLESIAS et. al., 2005; MEMMERT; GRECO; MORALES, 2010; MORALES; GRECO, 2007; MORALES; ANDRADE; GRECO, 2009) têm utilizado os instrumentos desenvolvidos por French e Thomas (1987), por Oslin, Mitchell e Griffin (1998) e por Memmert (2002). Contudo, observa-se que tais instrumentos, mesmo apresentando categorias comuns aos jogos esportivos coletivos, não avaliam os componentes táticos específicos do jogo do basquetebol.

O objetivo deste estudo foi estabelecer a validade de conteúdo do teste de conhecimento tático processual para basquetebol (TCTP-Bb), a partir do cálculo do coeficiente de validade de conteúdo (CVC) proposto por Hernandez-Nieto (2002).

\section{Método}

Este estudo foi aprovado pelo Comitê de Ética em Pesquisa (COEP) sob o protocolo CAAE - 0290.0.203.000-11. Participaram voluntariamente sete técnicos de basquetebol $(\mathrm{n}=07)$ formados em educação física e com experiência mínima de dez anos atuando especificamente no processo de iniciaçáo esportiva, especialização e alto nível de rendimento na modalidade.

O TCTP - Bb pretende avaliar o comportamento táticotécnico individual e de grupo do jogador no ataque e na defesa, a partir de uma situação de igualdade numérica (3x3) em campo reduzido. Nesse contexto, a construçáo do teste seguiu o modelo para elaboração de instrumentos psicométricos proposto por Pasquali (2010), no que se refere aos procedimentos teóricos (análise semântica dos itens e análise dos juízes).

Definiram-se como inerentes ao sistema psicológico os processos cognitivos relacionados à tomada de decisão nos JEC. Sua propriedade define-se pelo nível de conhecimento tático processual (CTP) que o atleta possui sendo o objeto psicológico a tomada de decisão do atleta. O sistema psicológico foi dividido em duas dimensóes denominadas de ataque e defesa.
$\mathrm{Na}$ análise semântica do instrumento, foram propostos vinte e quatro (24) critérios de observaçáo (itens) para avaliar o comportamento tático - técnico individual e de grupo do jogador no ataque (sem posse de bola e com posse de bola) e na defesa (marcando ao jogador sem posse de bola e com posse de bola), em situação de igualdade numérica (3x3) em espaço reduzido.

Para análise teórica dos 24 itens do instrumento utilizouse a técnica de validade de conteúdo, no que se refere à clareza da linguagem e a pertinência prática. Calculou-se o coeficiente de validade de conteúdo (CVC) proposto por HernandezNieto, (2002), para cada item do instrumento $\left(\mathrm{CVC}_{\mathrm{c}}\right)$ e para o instrumento como um todo ( $\mathrm{CVC}_{\mathrm{t}}$ ), assim os juízes utilizaram uma escala de 01 a 05 pontos para avaliar o nível de adequação da clareza da linguagem e da pertinência prática dos 24 itens propostos Hernandez-Nieto, (2002).

1) com base nas notas dos juízes, calculou-se a média das notas de cada item conforme proposto por HernandezNieto (2002) $(M x)$ :

$$
M_{x}=\frac{\sum_{i=1}^{J} x_{i}}{J}
$$

Onde $\sum x i$ representa a soma das notas dos juízes e $J$ representa o número de juízes que avaliaram o item.

2) Com base na média, calculou-se o $C V C$ para cada item $(C V C i)$ :

$$
C V C_{i}=\frac{M_{x}}{V_{\text {máx }}}
$$

Onde Vmáx representa o valor máximo que o item poderia receber.

3) Realizou-se ainda o cálculo do erro $\left(\mathrm{P}_{\mathrm{ei}}\right)$, para descontar possíveis vieses dos juízes avaliadores, para cada item:

$$
P e_{i}=\left(\frac{1}{J}\right)^{J}
$$

4) Com isso, o $C V C$ final de cada item $(C V C c)$ foi assim calculado:

$$
C V C_{c}=C V C_{i}-P e_{i}
$$

5) Para o cálculo do $C V C$ total do questionário $(C V C t)$, para cada uma das características (clareza de linguagem e pertinência prática), utilizou-se: 


$$
C V C_{t}=M c v c_{i}-M p e_{i}
$$

Onde Mcvci representa a média dos coeficientes de validade de conteúdo dos itens do questionário e $M p e i$, a média dos erros dos itens do questionário.

O ponto de corte adotado para determinar níveis satisfatórios para clareza da linguagem e pertinência foi de $\mathrm{CVC}_{\mathrm{c}} \geq 0,70$ para cada um dos itens e de $\mathrm{CVC}_{\mathrm{t}} \geq 0,70$ para o instrumento no geral conforme recomendado na literatura da área (CASSEPP-BORGES; BALBINOTTI; TEODORO, 2010).

\section{Resultados}

Em relação à clareza da linguagem, observa-se na Tabela 01 que o instrumento apresentou um $\mathrm{CVC}_{\mathrm{t}}=0,94$, acima do ponto de corte estabelecido. Dos 24 itens propostos o item 16 apresentou um CVC abaixo do ponto de corte $\left(\mathrm{CVC}_{\mathrm{c}}=\right.$ 0,68). Nesse contexto, Hernandez-Nieto (2002) propóe que para clareza de linguagem os itens com um $\mathrm{CVC}_{\mathrm{c}}$ abaixo do ponto de corte estabelecido sejam reformulados e encaminhados novamente para serem avaliados pelos juízes. No entanto, esse procedimento náo foi adotado devido a que esse mesmo item (16) apresentou para pertinência prática um $\mathrm{CVC}_{\mathrm{c}}$ abaixo do ponte de corte.

TABELA 1. Cálculo do CVC para clareza da linguagem do TCTP-Bb

\begin{tabular}{ccccc}
\hline ITEM & MÉDIA & CVCi & Pei & CVCc \\
\hline 1 & 5 & 1 & 0,00000121 & 0,999999 \\
2 & 5 & 1 & 0,00000121 & 0,999999 \\
\hline 3 & 5 & 1 & 0,00000121 & 0,999999 \\
4 & 5 & 1 & 0,00000121 & 0,999999 \\
\hline 5 & 5 & 1 & 0,00000121 & 0,999999 \\
\hline 6 & 5 & 1 & 0,00000121 & 0,999999 \\
\hline 7 & 5 & 1 & 0,00000121 & 0,999999 \\
\hline 8 & 4,428571 & 0,885714 & 0,00000121 & 0,885713 \\
\hline 9 & 4,571429 & 0,914286 & 0,00000121 & 0,914285 \\
\hline 10 & 4,428571 & 0,885714 & 0,00000121 & 0,885713 \\
\hline 11 & 4,714286 & 0,942857 & 0,00000121 & 0,942856 \\
\hline 12 & 4,714286 & 0,942857 & 0,00000121 & 0,942856 \\
\hline 13 & 5 & 1 & 0,00000121 & 0,999999 \\
\hline 14 & 4,428571 & 0,885714 & 0,00000121 & 0,885713 \\
\hline 15 & 4,714286 & 0,942857 & 0,00000121 & 0,942856 \\
\hline 16 & 3,428571 & 0,685714 & 0,00000121 & 0,685713 \\
\hline 17 & 4,285714 & 0,857143 & 0,00000121 & 0,857142 \\
\hline 18 & 5 & 1 & 0,00000121 & 0,999999 \\
\hline 19 & 5 & 1 & 0,00000121 & 0,999999 \\
\hline & & & &
\end{tabular}

\begin{tabular}{ccccc}
\hline ITEM & MÉDIA & CVCi & Pei & CVCc \\
\hline 20 & 5 & 1 & 0,00000121 & 0,999999 \\
21 & 5 & 1 & 0,00000121 & 0,999999 \\
22 & 4,142857 & 0,828571 & 0,00000121 & 0,82857 \\
23 & 4,428571 & 0,885714 & 0,00000121 & 0,885713 \\
24 & 4,857143 & 0,971429 & 0,00000121 & 0,971427 \\
CVCt & & & & $\mathbf{0 , 9 4 2 8 5 6}$ \\
\hline
\end{tabular}

Em relação à pertinência prática o instrumento apresentou um $\mathrm{CVC}_{\mathrm{t}}=0,91$, acima do ponto de corte estabelecido (TABELA 2). Novamente o item 16 (em formação de triângulo, dois jogadores se deslocam em direção de um terceiro que recebeu passe e procuram se librar da marcação), proposto para avaliar o comportamento tático-técnico de grupo no ataque, foi o único que apresentou um CVC abaixo do ponto de corte $\left(\mathrm{CVC}_{\mathrm{c}}=0,65\right)$. Nesse contexto, optou-se por descartar esse item do instrumento.

TABELA 2. Cálculo do CVC para pertinência prática do TCTP-Bb

\begin{tabular}{|c|c|c|c|c|}
\hline ITEM & MÉDIA & $\mathrm{CVCi}$ & Pei & CVCc \\
\hline 1 & 4,857143 & 0,971429 & 0,00000121 & 0,971427 \\
\hline 2 & 4,714286 & 0,942857 & 0,00000121 & 0,942856 \\
\hline 3 & 3,857143 & 0,771429 & 0,00000121 & 0,771427 \\
\hline 4 & 3,857143 & 0,771429 & 0,00000121 & 0,771427 \\
\hline 5 & 4,857143 & 0,971429 & 0,00000121 & 0,971427 \\
\hline 6 & 4,833333 & 0,966667 & 0,00000121 & 0,966665 \\
\hline 7 & 4,833333 & 0,966667 & 0,00000121 & 0,966665 \\
\hline 8 & 4,5 & 0,9 & 0,00000121 & 0,899999 \\
\hline 9 & 4,666667 & 0,933333 & 0,00000121 & 0,933332 \\
\hline 10 & 4,5 & 0,9 & 0,00000121 & 0,899999 \\
\hline 11 & 5 & 1 & 0,00000121 & 0,999999 \\
\hline 12 & 5 & 1 & 0,00000121 & 0,999999 \\
\hline 13 & 5 & 1 & 0,00000121 & 0,999999 \\
\hline 14 & 5 & 1 & 0,00000121 & 0,999999 \\
\hline 15 & 4,857143 & 0,971429 & 0,00000121 & 0,971427 \\
\hline 16 & 3,285714 & 0,657143 & 0,00000121 & 0,657142 \\
\hline 17 & 3,714286 & 0,742857 & 0,00000121 & 0,742856 \\
\hline 18 & 4,857143 & 0,971429 & 0,00000121 & 0,971427 \\
\hline 19 & 4,571429 & 0,914286 & 0,00000121 & 0,914285 \\
\hline 20 & 4,857143 & 0,971429 & 0,00000121 & 0,971427 \\
\hline 21 & 4,571429 & 0,914286 & 0,00000121 & 0,914285 \\
\hline 22 & 4,571429 & 0,914286 & 0,00000121 & 0,914285 \\
\hline 23 & 4,571429 & 0,914286 & 0,00000121 & 0,914285 \\
\hline 24 & 4,857143 & 0,971429 & 0,00000121 & 0,971427 \\
\hline CVCt & & & & 0,918253 \\
\hline
\end{tabular}


Finalmente, todos os itens foram ajustados conforme as sugestóes dos juízes e o processo de validade de conteúdo, por meio do cálculo do CVC (HERNADEZ-NIETO, 2002), determinou um total de 23 itens para compor a versão inicial do TCTP - Bb (QUADRO 01).

QUADRO 01. Itens que compóem a versão inicial do TCTP-Bb após o cálculo do CVC para clareza da linguagem e pertinência prática

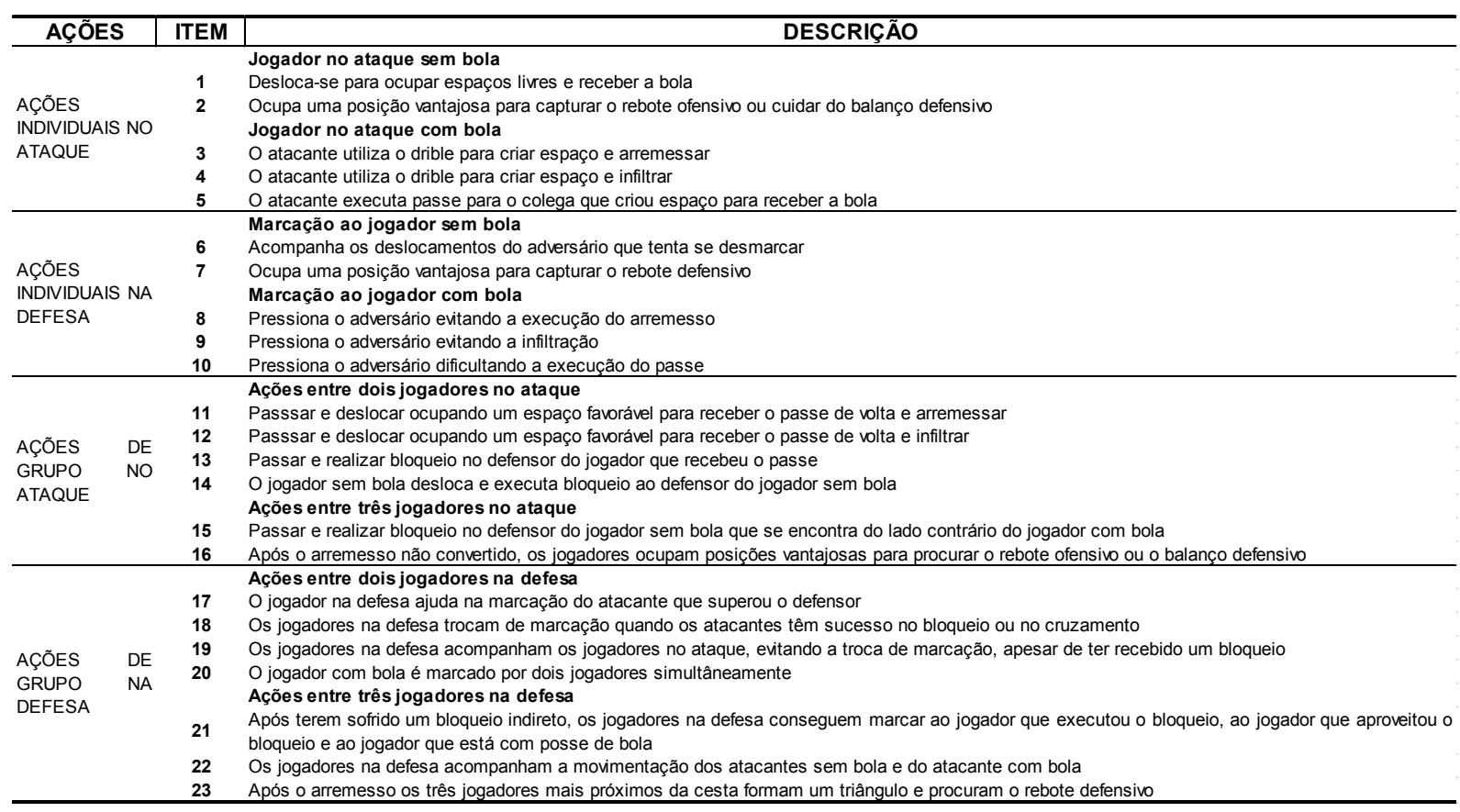

\section{Discussão}

Pode-se afirmar que o CVC calculado para clareza da linguagem $\left(\mathrm{CVC}_{\mathrm{t}}=0,94\right)$ e pertinência prática $\left(\mathrm{CVC}_{\mathrm{t}}=0,91\right)$ do TCTP - Bb é satisfatório, pois está acima do ponto de corte sugerido por Hernandez-Nieto (2002).

No estudo de Valentini et. al. (2008) o CVC calculado para clareza da linguagem do teste de desenvolvimento motor grosso foi de $\mathrm{CVC}_{t}=0,96$ e para pertinência prática foi de $\mathrm{CVC}_{\mathrm{t}}=0,96$.

No estudo de Aburachid e Greco (2010), o processo de validade de conteúdo do teste de conhecimento tático para o tênis determinou um $\mathrm{CVC}_{\mathrm{t}}=0,89$ para clareza e um $\mathrm{CVC}_{\mathrm{t}}=$ 0,91 para pertinência.

$\mathrm{Na}$ escala para avaliação do conhecimento tático processual nos jogos esportivos coletivos de invasão proposta por Morales, Aburachid e Greco (2011a) para o futebol, o cálculo do CVC para clareza da linguagem foi de $\mathrm{CVC}_{t}=0,81$ e para pertinência prática foi de $\mathrm{CVC}_{t}=0,80$. Essa mesma escala aplicada no futsal (MORALES; ABURACHID; GRECO, 2011b) alcançou um $\mathrm{CVC}_{\mathrm{t}}=0,81$ para clareza da linguagem $\mathrm{e}$ de $\mathrm{CVC}_{\mathrm{t}}=0,87$ para pertinência prática. No handebol (MORALES; ABURACHID; GRECO, 2011c) os valores calculados foram de $\mathrm{CVC}_{\mathrm{t}}=0,80$ para clareza da linguagem e de $\mathrm{CVC}_{\mathrm{t}}=0,94$ para pertinência prática.

No processo de validaçáo do teste de coordenaçáo com bola (TECOBOL), proposto por Silva (2011), os valores calculados para clareza da linguagem foram $\mathrm{CVC}_{t}=0,93$, para relevância teórica de $\mathrm{CVC}_{\mathrm{t}}=0,99$ e para representatividade obtiveram-se $\mathrm{CVC}_{\mathrm{t}}=0,90$ respectivamente.

Os valores do CVC calculados neste estudo para clareza da linguagem e pertinência prática estão dentro dos parâmetros são aceitos pela literatura. Pode-se consequentemente afirmar que os itens propostos para analisar o comportamento tático-técnico individual e de grupo em jogadores de basquetebol, por meio de uma situação de igualdade numérica (3x3) e utilizando campo reduzido são adequados. 


\section{Referências}

Aburachid, C.L.M. \& Greco, P.J. (2010). Processos de validação de um teste de conhecimento tático declarativo no tênis. Revista Brasileira de Cineantropometria \& Desempenho Motor, 21(4), 603-610.

Allison, S. \& Thorpe, R. (1997). A comparison of the effectiveness of two pproaches to teaching games within physical education. A Skills approach versus a Games for Understanding approach. The British Journal of Physical Education, 28(3), 9-13.

Boutmans, J. (1983). Comparative effectiveness of two methods of teachings team sports in secundary schoools. In: Congresso AIESEP. Teaching Team Sports, 239-247.

Cassepp-Borges, V., Balbinotti, M.A.A. \& Teodoro, M.L.M. (2010). Tradução e validação de conteúdo: uma proposta para adaptação de instrumentos. In: Pasquali, L. (Org.), Instrumentaçâo psicológica. Fundamentos e práticas (pp.506-520). Porto Alegre: Artmed.

Costa, I.; Garganta, J.; Greco, P.; Mesquita, I. \& Maia, J. (2011). Sistema de avaliação tática no futebol. Motricidade, 7(1), 69-84.

French, K.E. \& Thomas, J. (1987). The relation of knowledge development to children's basketball performance. Journal of Sport Psychology, 9, 1532 .

Garganta, J. (2004). A formação estratégico - tática nos jogos desportivos de oposição e cooperação. In: Gaya, A.; Marques, A. \& Tani, G. (Org.) Desporto para crianças e jovens. Razóes e finalidades (pp. 217-233). Porto Alegre: Editora UFRGS.

Greco, P.J. (1999). Cognição e Ação. In: Samulski, D. (Ed.) Novos Conceitos em Treinamento Esportivo (pp. 119-153). Belo Horizonte: Publicações Indesp.

Greco, P.; Memmert, D. \& Morales, J. C. (2010). The effect of deliberate play on tactical performance in basketball. Perceptual Motor Skills, $110(3), 849-56$

Gréhaigne, J. F.; Godbout, P. \& Bouthier, D. (1997). Performance assessment in team sports. Journal of Teaching in Physical Education, 16(4), 500-516.

Gréhaigne, J. F.; Godbout, P. \& Bouthier, D. (2001). The teaching and learning of decision making in team sports. Quest, 53 (1), 59-76.

Hernández-Nieto, R. A. (2002). Contributions to Statistical Analysis (pp. 119). Mérida: Universidad de Los Andes.

Iglesias, D.; Sanz, D.; García, T.; Cervelló, E.M. \& Del Villar, F. (2005). Influencia de un programa de supervision reflexive sobre la toma de decisiones y la ejecución del pase en jóvenes jugadores de baloncesto. Revista de Psicologia del Deporte, 14(2), 209-223.

Memmert, D. (2002). Diagnostik Taktischer Leistungskomponenten: Spieltestsituationen und Konzeptorientierte Expertenratings. Tese (Doutorado) Universidade de Heidelberg, Heidelberg.

Morales, J.C.P.; Aburachid, L.M.C. \& Greco, P.J. (2011a). Escala para avaliaçáo do conhecimento tático processual nos jogos esportivos coletivos de invasão: validação do conteúdo no futebol. Revista Portuguesa de Ciências do Desporto, 11(supp.4), 70.
Morales, J.C.P.; Aburachid, L.M.C. \& Greco, P.J. (2011b). Escala para avaliação do conhecimento tático processual nos jogos esportivos coletivos de invasão: validação do conteúdo no futsal. Revista Portuguesa de Ciências do Desporto, 11(supp.4), 71.

Morales, J.C.P.; Aburachid, L.M.C. \& Greco, P.J. (2011c). Escala para avaliação do conhecimento tático processual nos jogos esportivos coletivos de invasão: validação do conteúdo no handebol. Revista Portuguesa de Ciências do Desporto, 11(supp.4), 71.

Morales, J.C.P., \& Greco, P.J. (2007). A influência de diferentes metodologias de ensino-aprendizagem-treinamento no basquetebol sobre o nível de conhecimento tático processual. Revista Brasileira de Educação Física e Esporte, 21(4), 291-299.

Morales, J.C.P; Andrade, R.L. \& Greco, P.J. (2019). A description of the teaching-learning processes in basketball and their effects on tactical procedural knowledge. Revista de Psicologia del Deporte, 18 - suppl., 469-473.

Nitsch, J.R. (2009). Ecological approaches to Sport Activity: A commentary from an action-theoretical point of view. International Journal Sport Psychology, 40, 152-176.

Oslin, j. L.; Mitchell, S. A. \& Griffin, L. L. (1998). The Game Performance Assessment Instrument (GPAI): development and preliminary validation. Journal of Teaching in Physical Education, 17(2), 231-243.

Pasquali, L. (2010). Testes referentes a construto: teoria e modelo de construção. In: Pasquali, L. (Org.) Instrumentação Psicológica. Fundamentos e Práticas (pp. 165-198). Porto Alegre: Artmed.

Rodrigues, J.A. (2001). A capacidade de decisão tática e o conhecimento do jogo em jogadores juniores de basquetebol. In: Tavares, F., Janeira, M., Graça, A., Pinto, D. \& Brandão, E. (Eds.), Tendências Actuais da investigação em basquetebol (pp. 227-234). Porto: Universidade do Porto. Faculdade de Ciências do Desporto e de Educação Física.

Silva, S. (2011). Manual do teste de coordenação com bola - TECOBOL. Revista Portuguesa de Ciências do Desporto, 11(supp.4), 92.

Sève, C.; Saury, j.; Leblanc, S. \& Durand, M. (2005). Course-of-action theory in table tennis: a qualitative analysis of the knowledge used by three elite players during matches. Revue européenne de psychologie appliquée. 55, 145-155.

Tallir, I. B.; Musch, E.; Kristof, L. \& Voorde, J. V. D. (2003). Validation of a video-based coding instrument for the assessment of invasion games competence as a player in handball and soccer. In: 2nd International Conference: teaching sport and physical education for understanding (pp.44). Melbourne: University of Melbourne, Australia.

Valentini, N.C.; Barbosa, M.L.L; Cini, G.V.; Pick, R.K.; Spessato, B.C. \& Balbinotti, M.A.A. (2008). Teste de desenvolvimento motor grosso: validade e consistência interna para uma população gaúcha. Revista Brasileira de Cineantropometria \& Desempenho Motor, 10(4), 399-404. 